\title{
Persistent Circulation Regimes and Preferred Regime Transitions in the North Atlantic
}

\author{
CHRISTIAN FRANZKE \\ British Antarctic Survey, Cambridge, United Kingdom \\ TIM WOOLLINGS \\ Department of Meteorology, University of Reading, Reading, United Kingdom \\ OLIVIA MARTIUS \\ Oeschger Center for Climate Change Research and Institute of Geography, University of Bern, Bern, Switzerland
}

(Manuscript received 31 January 2011, in final form 27 June 2011)

\begin{abstract}
The persistent regime behavior of the eddy-driven jet stream over the North Atlantic is investigated. The North Atlantic jet stream variability is characterized by the latitude of the maximum lower tropospheric wind speed of the 40-yr ECMWF Re-Analysis (ERA-40) data for the period 1 December 1957-28 February 2002. A hidden Markov model (HMM) analysis reveals that the jet stream exhibits three persistent regimes that correspond to northern, southern, and central jet states. The regime states are closely related to the North Atlantic Oscillation and the eastern Atlantic teleconnection pattern. The regime states are associated with distinct changes in the storm tracks and the frequency of occurrence of cyclonic and anticyclonic Rossby wave breaking. Three preferred regime transitions are identified, namely, southern to central jet, northern to southern jet, and central to northern jet. The preferred transitions can be interpreted as a preference for poleward propagation of the jet, but with the southern jet state entered via a dramatic shift from the northern state. Evidence is found that wave breaking is involved in two of the three preferred transitions (northern to southern jet and central to northern jet transitions). The predictability characteristics and the interannual variability in the frequency of occurrence of regimes are also discussed.
\end{abstract}

\section{Introduction}

Atmospheric circulation regimes are preferred states of the atmosphere with a high probability of occurrence or a high degree of persistence and are thus important for predictability. Atmospheric regimes are commonly used to categorize the continuum of atmospheric flows into discrete circulation patterns. These regimes are the imprint of nonlinear interactions across many scales (Majda et al. 2006; Franzke et al. 2007) and their existence can lead to non-Gaussian statistics (Kimoto and Ghil 1993; Corti et al. 1999; Smyth et al. 1999; Berner and Branstator 2007), although Majda et al. (2006)

Corresponding author address: Dr. C. Franzke, British Antarctic Survey, High Cross, Madingley Road, Cambridge CB3 0ET, United Kingdom.

E-mail: chan1@bas.ac.uk showed the existence of regimes in systems with unimodal, nearly Gaussian statistics.

The pioneering study by Charney and DeVore (1979) first introduced the notion of flow regimes and their connection to blocking in a very low-order reduced barotropic model of flow over topography. In this severely truncated model, Charney and DeVore (1979) found that the two regime states, zonal flow and blocking, correspond to two fixed points. Wiin-Nielsen (1979) and Legras and Ghil (1985) found similar results. Contrary to these studies, Reinhold and Pierrehumbert (1982), Tung and Rosenthal (1985), Cehelsky and Tung (1987), and Majda et al. (2006) found that the distinct flow regimes are not close to the fixed points of the truncated planetary waves in models with more resolved waves. These studies suggest that the full spectrum of waves cannot be neglected and affects the characteristics of circulation regimes. 
Besides these dynamically motivated studies many statistical studies have been carried out (Mo and Ghil 1988; Cheng and Wallace 1993; Kimoto and Ghil 1993; Corti et al. 1999; Smyth et al. 1999; Monahan et al. 2001, 2003; Kondrashov et al. 2004; Kravtsov et al. 2005; Berner and Branstator 2007; Woollings et al. 2010). These studies usually take a static point of view by searching for preferred patterns or preferred locations in phase space. Temporal information is often neglected, although some studies try to estimate a posteriori the transition probabilities between the regime states by assigning each data point to a regime depending on its location in phase space (Kimoto and Ghil 1993; Kondrashov et al. 2004; Crommelin 2004).

An important question from a predictability point of view is if the states with the highest probability of occurrence, so-called recurrent states, are also persistent states. The probability density function (PDF) only gives information how often a specific circulation pattern occurs. The PDF does not tell us if the circulation tends to stay in this region of phase space for a long time or just passes quickly through this phase space region and returns very often. Persistent circulation states offer the potential of enhanced predictability. The study by Vautard (1990) looks for persistent atmospheric circulation states by minimizing the tendency of $700-\mathrm{hPa}$ geopotential height fields. However, Michelangeli et al. (1995) show that the persistent states found by Vautard (1990) do not necessarily correspond to recurrent states. Michelangeli et al. (1995) examine the geopotential height tendencies of the recurrent states. They find that the observed tendencies of the recurrent regimes are strong enough to alter the geographical structure of the recurrent regime patterns within a few days. Hence in their study the recurrent regimes do not correspond to persistent regimes, because of their strong tendencies. Furthermore, Michelangeli et al. (1995) show that the centroids $^{1}$ of many recurrent regimes do not correspond to the location of the quasi-stationary regimes.

From a predictability perspective it is interesting to identify the patterns that are recurrent as well as persistent. Such patterns offer the most potential to increase predictability. Systematic clustering approaches have been used to identify persistent regimes. They decompose the phase space into preferred locations while simultaneously requiring persistence, or metastability, of the resulting regime states (Majda et al. 2006; Franzke et al. 2008, 2009; Horenko 2008a, 2010; Horenko et al. 2008). Such approaches have been tested in a hierarchy of circulation models (Majda et al. 2006; Franzke et al.

\footnotetext{
${ }^{1}$ A centroid denotes the center of a regime in phase space.
}

2008, 2009) and reanalysis data (Horenko et al. 2008; Horenko 2010).

An important topic from a predictability perspective is the identification of preferred transitions between the regimes. The underlying idea of regimes is that all circulation states can be assigned to a finite number of regime states. The continuous change of the atmospheric circulation can now be seen as discrete transitions between the regimes. In this view, while the atmospheric circulation is always changing, it can still belong to a regime for a long period of time; we refer to this as persistence. But after some time it will transition to another regime. Now the question is: are the transitions to all other regimes equally likely or are some transitions much more likely? Transitions are called preferred transitions if they are statistically significantly more likely than if all transitions were equally likely. The discretization into regime states allows us to fit a Markov transition matrix to the regime transitions (Kimoto and Ghil 1993; Kondrashov et al. 2004, 2007; Crommelin 2004). The clustering method used in Majda et al. (2006) and Franzke et al. (2008) simultaneously infers the preferred patterns, their Markov transition probabilities, and the most likely evolution of the atmospheric circulation among the regime states.

In this study we investigate the regime characteristics of the North Atlantic jet stream. We will examine if the previously identified regime states by Woollings et al. (2010) are persistent by applying the hidden Markov model (HMM) method (Rabiner 1989; Majda et al. 2006; Franzke et al. 2008). We will look for the existence of preferred transitions between the regimes and will examine the predictability properties of the regimes. We also investigate the link among synoptic-scale waves, Rossby wave breaking, and the regimes. Furthermore, we also examine the annual and interannual variability of the regimes. In previous studies the impact of eddy feedback on the persistence of the zonal jet has been highlighted (Branstator 1995; Lorenz and Hartmann 2003; Robinson 2006; Gerber and Vallis 2007). Because of the strong link between eddy feedback and wave breaking we want to examine how wave breaking affects the regime behavior. There are two dominant forms of wave breaking: anticyclonic (LC1) and cyclonic (LC2) wave breaking. ${ }^{2}$ Hartmann and Zuercher (1998) showed that the sharp transition from anticyclonic to cyclonic wave breaking in idealized baroclinic eddy life cycle experiments is due to the strong feedback between eddy

\footnotetext{
${ }^{2}$ Following Thorncroft et al. (1993), cyclonic wave breaking is characterized by southeast-northwest tilt of the trough-ridge pair. Anticyclonic wave breaking exhibits the opposite tilt.
} 
propagation and the zonal flow. Such a feedback is a potential candidate for causing persistent regime behavior of the Atlantic eddy-driven jet stream. This motivates us to investigate the wave breaking characteristics of the North Atlantic jet stream regimes.

The Jet Latitude Index (JLI) is described in section 2, the HMM methodology is briefly described in section 3, section 4 describes the regime states, sections 5 and 6 respectively discuss the preferred transitions among the regime states and their predictability, and section 7 discusses their intra- and interannual variability. A summary of our results is provided in section 8 .

\section{Data}

The Jet Latitude Index is a measure of the variability of the eddy-driven jet stream over the North Atlantic (Woollings et al. 2010; Franzke and Woollings 2011). Because the atmospheric circulation of the North Atlantic is closely linked to the eddy-driven North Atlantic jet stream and the same eddies are intimately linked to the dominant modes of variability in the North Atlantic region such as the North Atlantic Oscillation (NAO) (Benedict et al. 2004; Franzke et al. 2004; Martius et al. 2007; Woollings et al. 2008), the JLI provides an excellent measure of North Atlantic climate variability and its regime characteristics.

The JLI covers the period 1 December 1957-28 February 2002 and is derived in the following way from the 40-yr European Centre for Medium-Range Weather Forecasts (ECMWF) Re-Analysis (ERA-40) reanalysis data (Uppala et al. 2005): (i) a mass-weighted average of the daily mean zonal wind is taken over the vertical levels $925,850,775$, and $700 \mathrm{hPa}$ and over the Atlantic sector $0^{\circ}-60^{\circ} \mathrm{W}$. (ii) Winds poleward of $75^{\circ} \mathrm{N}$ and equatorward of $15^{\circ} \mathrm{N}$ are neglected. (iii) The resulting wind fields are low-pass filtered by only retaining periods greater than 10 days. (iv) The JLI is defined as the latitude at which the maximum wind speed is found. (v) A smooth seasonal cycle is subtracted from the resulting time series. See Woollings et al. (2010) for more details. In keeping with its definition, this index is a discrete valued index because only a finite number of latitudinal values are allowed, given the finite horizontal resolution of the ERA-40 reanalysis data. This index is strongly connected to the NAO and the East Atlantic (EA) teleconnection pattern as shown by Woollings et al. (2010).

Synoptic-scale Rossby wave breaking (RWB) events are identified in the ERA-40 dataset on the 310-360-K isentropic level with 5-K intervals following the method of Wernli and Sprenger (2007) and stratified according to their life cycle [cyclonic (LC2) or anticyclonic (LC1); see Martius et al. (2007) for more details]. The output of the identification algorithm is binary fields indicating the presence or absence of a RWB event for every 6-h time step on a $1^{\circ} \times 1^{\circ}$ geographical grid. The height of isentropic surfaces varies significantly with the season and a vertical integration of the fields is therefore necessary for a continuous analysis. For each time step all grid points where a potential vorticity (PV) streamer is detected on any vertical level are set to one. The resulting composites therefore only provide frequency information that is useful for a relative comparison of the different jet states but that cannot be readily transferred into absolute frequency values.

We also use 500-hPa geopotential height fields from the ERA-40 reanalysis data. We use a digital Lanczos filter with 31 weights to derive high-pass (periods less than 10 days retained) and low-pass (periods larger than 10 days retained) filtered data. A smooth seasonal cycle is subtracted from these fields [see Franzke and Feldstein (2005) for details].

\section{Systematic regime identification by hidden Markov models}

In a study by Woollings et al. (2010) evidence was found for regime behavior of the JLI in the winter season by analyzing the probability density functions of the JLI and the related NAO and EA indices. Such preferred states can mean that the JLI index visits these regions just very often for short periods of time. The alternative possibility is that the JLI stays in certain regions of phase space for long periods of time, suggesting a tendency for persistence and potentially enhanced predictability. Both possibilities would represent very interesting imprints of nonlinear behavior of the climate system, and it is also important to distinguish between them. Here we want to test if the preferred states of the JLI are persistent. For this purpose we follow the hidden Markov model approach of Majda et al. (2006) and Franzke et al. (2008) and apply it to the JLI.

The HMM method is able to extract persistent circulation regimes in a systematic fashion as shown in Majda et al. (2006) and Franzke et al. (2008). [See the appendixes for a detailed brief discussion of the HMM method. More details are given in Majda et al. (2006), Franzke et al. (2008), and Rabiner (1989).] The idea behind using HMM for regime identification is that a low-frequency process exists that governs the transitions between the regime states. It is assumed that this low-frequency process, which may have quite complex dynamics associated with it, can be approximately represented by a Markov chain. Here the regime states determine the characteristics of the observed jet (e.g., latitude, speed, variability) that are different and distinct for every regime state and 
that can be directly observed (e.g., on weather maps). The transitions among the regime states occur on a much slower time scale than the typical day-to-day jet stream fluctuations. Because the HMM requires a continuous time series, we use the JLI for all seasons. As the JLI is not pattern based it can be easily calculated over all seasons without any complications arising from the changing of patterns during the annual cycle. Furthermore, we subtract a smooth annual cycle of the first moment. A power spectral analysis of the JLI shows that the first moment of the annual cycle has been effectively removed (not shown).

\section{Number of persistent regimes}

The fitting of HMMs to time series in itself does not provide information about the presence of persistent metastable regimes. To test for persistent regime behavior, one has to check if the Markov transition matrix that determines the evolution of the hidden variable $X$ shows signs of a significant gap in its eigenvalue spectrum (Majda et al. 2006; Franzke et al. 2008, 2009). As shown in Franzke et al. (2008), a gap in the eigenvalue spectrum indicates that the state space can be decomposed in two or more sets with relatively infrequent transitions between those sets. These sets are the persistent sets. To check if any gap is significant, we use a Monte Carlo approach similar to the one used in Franzke et al. (2008) (see appendixes for more details). The ratio $\operatorname{Re}\left(c_{2}\right) / \operatorname{Re}\left(c_{3}\right)$ is 0.884 ( $\operatorname{Re}$ denotes the real part of the eigenvalue) and smaller than the 10th percentile of the corresponding autoregressive moving average (ARMA) ensemble of 0.886 , the ratio $\operatorname{Re}\left(c_{3}\right) / \operatorname{Re}\left(c_{4}\right)$ is 0.559 and smaller than the 10th percentile of the corresponding ARMA ensemble of 0.934 , and finally the ratio $\operatorname{Re}\left(c_{4}\right) /\left(c_{5}\right)$ is 0.99 and larger than the 10th percentile of the corresponding ARMA ensemble of 0.911. Thus, this test reveals that there are three metastable regime states in the JLI that are statistically significant against the ARMA null model. This result is consistent with Woollings et al. (2010), who also find evidence for three regimes in the JLI in their wintertime analysis.

In Fig. 1 we display the PDF of the JLI together with the Gaussian approximations of the PDFs of the three hidden states from the HMM. The Gaussian mixtures are weighted according to the left eigenvector of the Markov transition matrix. The left eigenvector provides a measure of how much the individual Gaussian distributions associated with the hidden states (derived from HMM analysis) contribute to the observed PDF. It can be seen that one regime corresponds to the mode $^{3}$ of the

\footnotetext{
${ }^{3}$ The mode of a PDF denotes the maximum.
}

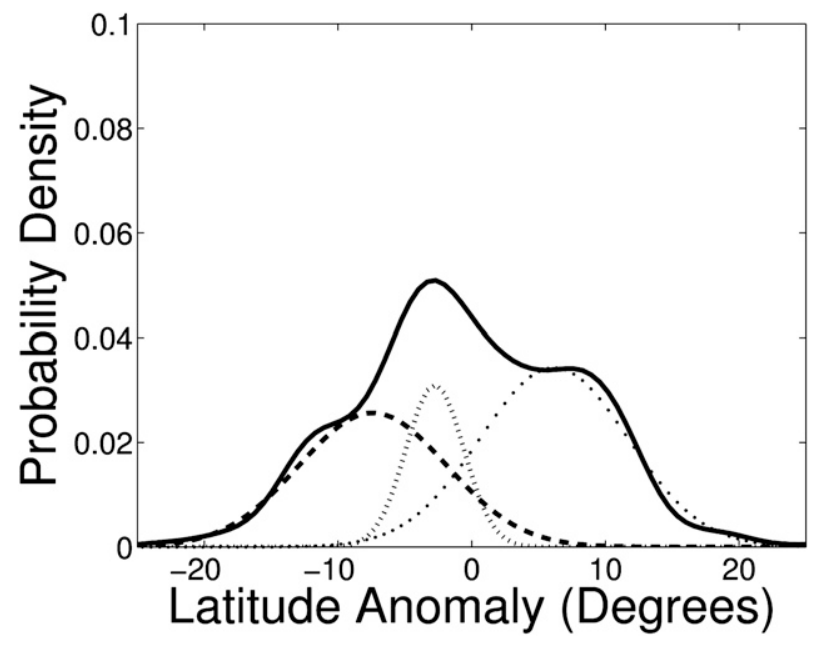

FIG. 1. PDF of the JLI (solid) together with the weighted Gaussian PDFs from the HMM: the southern (dashed), northern (dotted), and central (dashed-dotted) regimes. The three Gaussian PDFs only approximate the JLI PDF; thus, the sum of the three PDFs does not result in the JLI PDF.

PDF while the other two regimes correspond to the two shoulders of the PDF. Furthermore, the mode of the PDF is equatorward of the climatological mean state. This suggests that the climatological mean state is dynamically not very relevant and is thus a statistical artifact of the averaging procedure. A large overlap is visible of the Gaussian mixtures. A strong advantage of HMMs is not only that they allow for the overlap of the distributions but also that the classification of the current state to one of the hidden states takes into account the dynamics of the system (i.e., the metastability). In contrast, studies by Crommelin (2004) and Kondrashov et al. (2004) identify regime states by looking for maxima in the probability density function or by using Gaussian mixtures for regime identification. Thus, the regime states get strictly classified according to the likelihood of belonging to one of the partitions of the PDF (e.g., the Gaussian mixture components) (Kondrashov et al. 2004). In these approaches only a few bounded regions of the entire phase space are utilized and a state in phase space always belongs to the same unique regime (Crommelin 2004). In the HMM approach the temporal evolution of the system is taken into account in determining to which regime the current state belongs. At a later time the same state could belong to a different regime depending on the temporal evolution of the system.

Further evidence for persistent regime behavior is provided by the distribution of tendencies of the JLI in Fig. 2. The tendencies are calculated as $\widetilde{\mathrm{JLI}}(t+1)-\widetilde{\mathrm{JLI}}(t)$, where $\widetilde{\mathrm{JLI}}$ denotes a 3-day nonoverlapping mean. The striking feature of this distribution is the large kurtosis. This seems to reflect the persistent regime structure. There 


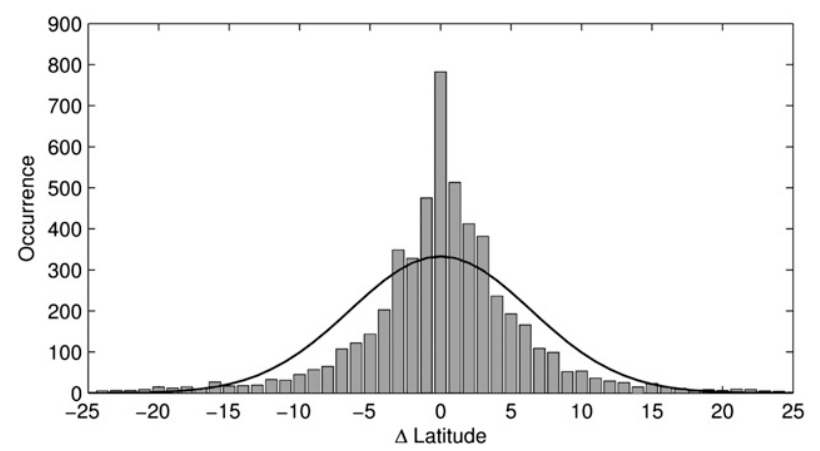

FIG. 2. Distribution of forward tendencies of 3-day mean JLI index values (vertical bars). The solid curve corresponds to a Gaussian distribution with the same mean and variance as the tendency distribution.

are many days with a very weak tendency, relatively few days of moderate tendencies, and enhanced tails of days with very large tendencies. The predominance of small tendencies can be interpreted as the imprint of the persistent regimes while the days with large tendencies can be interpreted as the regime transitions.

\section{North Atlantic circulation regimes}

\section{a. Geographical regime structure}

The metastable regime states are displayed in Fig. 3 in terms of $500-\mathrm{hPa}$ geopotential height anomalies with respect to the climatological mean. The regime states correspond to conditional averages (i.e., we average over all geopotential fields whenever the hidden state sequence is in state 1 , and so forth). The regime states correspond to a southern, northern, and central jet state and share many similarities with the wintertime states identified by Woollings et al. (2010). The southern jet state has a positive geopotential anomaly over Greenland and a negative anomaly over the central North Atlantic, thus leading to a southward shift of the jet stream. The northern jet regime state has just the

\section{a) Southern Jet}
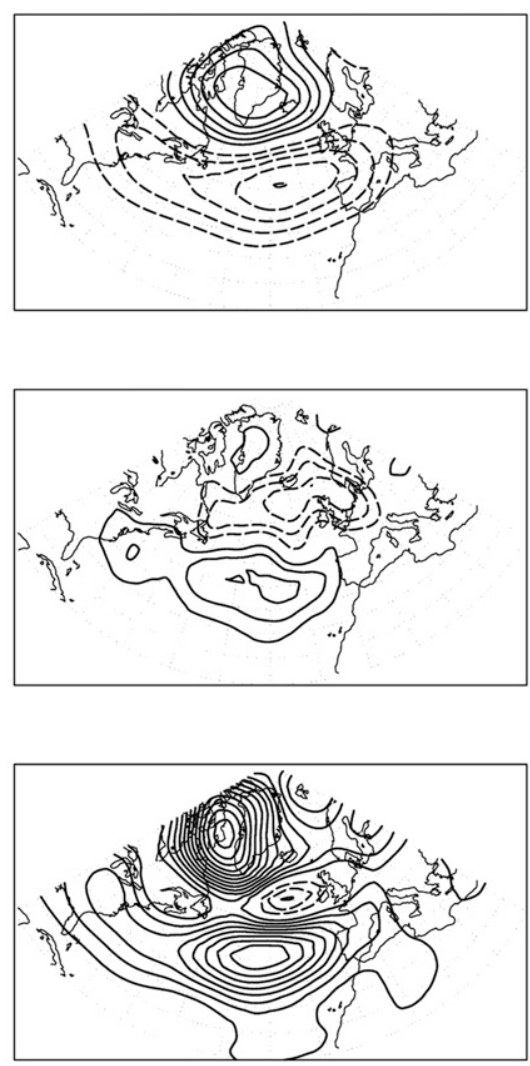

b) Northern Jet

Geopotential Height

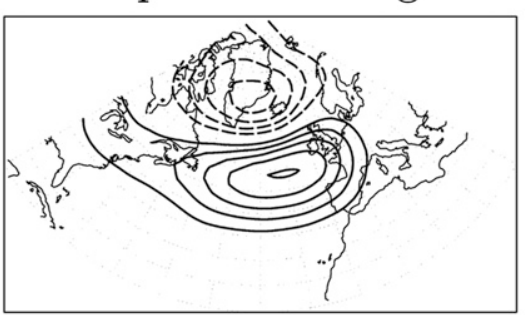

High-pass filtered variance

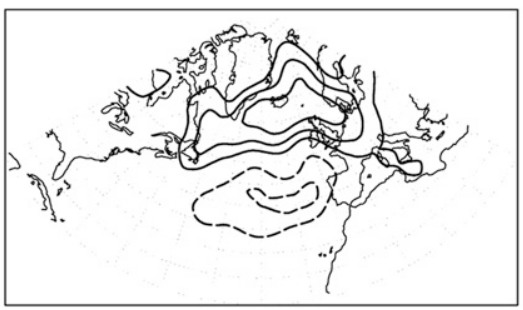

Low-pass filtered variance

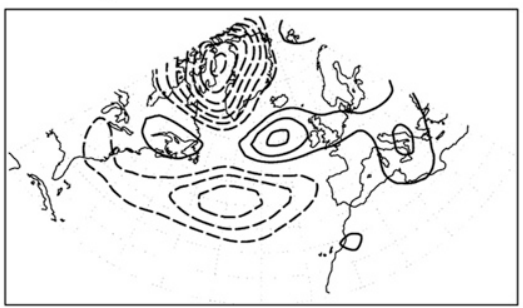

\section{c) Central Jet}
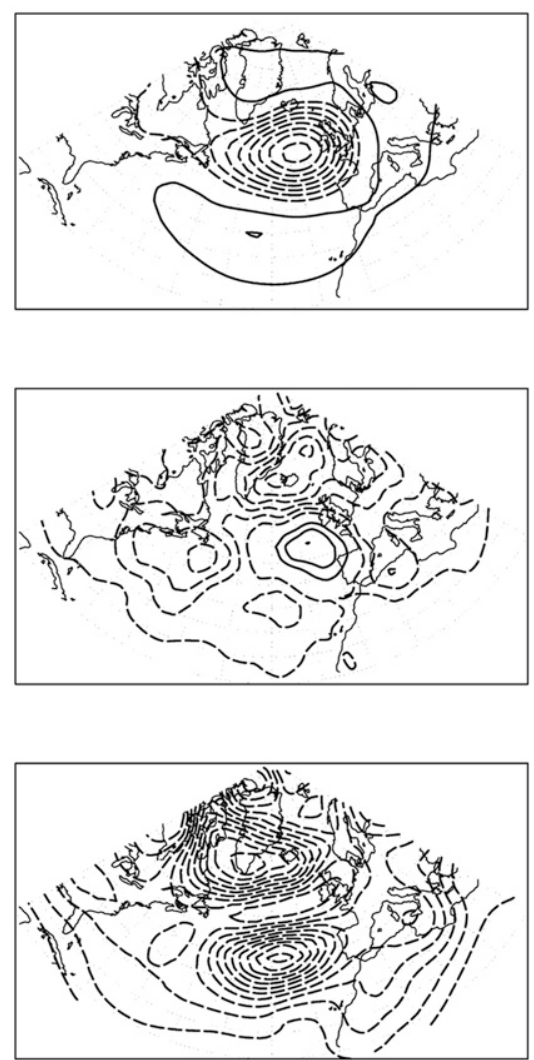

FIG. 3. (top) 500-hPa geopotential conditional mean fields with annual cycle subtracted for the three hidden states: (a) southern, (b) northern, and (c) central jets. Contour interval is $100 \mathrm{gpm}$. Also shown are the standard deviations of (middle) high- (periods less than 10 days) and (bottom) low-pass filtered (periods of more than 10 days) 500-hPa geopotential conditional mean fields for the three hidden states with climatological mean subtracted. Contour interval is $20 \mathrm{gpm}$. 
opposite polarity of the southern jet state and thus corresponds to a northward shift of the jet stream. The central jet regime state is associated with a negative monopole geopotential height anomaly over the central North Atlantic just west of the British Isles. The spatial patterns of the northern and southern jet regimes are very similar to the two phases of the NAO, and also the zonal and Greenland anticyclone regimes of Vautard (1990). The central jet does not resemble any of the previously identified Atlantic sector regimes. Furthermore, previous studies found evidence for four (Vautard 1990; Michelangeli et al. 1995; Cassou 2008) or even six (Kimoto and Ghil 1993) regimes in the North Atlantic sector. Two of our three regimes have very similar structures to the mid-Atlantic regimes in these previous studies. These studies also find a regime representing blocking over central Europe/Scandinavia. This regime does not appear in our analysis because we focus on Atlantic jet regimes and so analyze only data in the sector $0^{\circ}-60^{\circ} \mathrm{W}$ (Woollings et al. 2010). Our results are in fact consistent with the previous studies. While blocking over the Atlantic/western Europe is closely linked to the jet regimes, blocking over central Europe and Scandinavia is surprisingly independent of the Atlantic jet latitude and so can be considered a distinct flow regime (Woollings et al. 2010). It also has to be noted that the previous studies, despite finding more than three regimes, show some disagreement on the regime patterns.

Compositing the regime states according to season reveals very similar geographical structures but with weaker amplitude during summer than during winter (not shown). This is consistent with the findings of Barnston and Livezey (1987) that the NAO is the dominant teleconnection pattern not only during winter but also during summer. The study by Feldstein (2007) shows that the dynamics of the NAO are the same during winter and summer, as also shown by Woollings et al. (2010), and the NAO is closely related to the JLI.

\section{b. Regime imprint on storm tracks}

Furthermore, the regimes are associated with distinct changes in the storm tracks (variance of high-frequency fluctuations) and low-frequency variability. In the following we discuss deviations from the climatological variances (Fig. 3). In the southern jet state there is increased high-frequency eddy activity along the jet stream and reduced high-frequency eddy activity poleward of the jet stream. The low-frequency variability has maxima along the jet stream and over the eastern coast of Canada and Greenland and a minimum west of the British Isles. This might suggest increased blocking activity during the southern jet regime and is consistent with Rennert and Wallace (2009) and the blocking results of Woollings et al. (2010). For the northern jet regime the storm track has moved poleward and this regime state has a much reduced low-frequency variability over most of the North Atlantic but with enhanced low-frequency variability just west of the United Kingdom at the end of the jet stream. The central jet regime has increased high-frequency variability west of the Iberian Peninsula and the British Isles with reduced storm activity over most of the western and northern parts of the North Atlantic region. Also for this regime state the low-frequency variability is reduced over the North Atlantic region. Compositing the variance fields associated with the regime states according to season reveals again very similar geographical structures (not shown).

The low-frequency variability patterns (Fig. 3) are qualitatively similar to the blocking results by Scherrer et al. (2006) for the NAO and EA teleconnection patterns. As shown in Woollings et al. (2010), the jet regime states are closely related to the NAO and EA. ${ }^{4}$ The southern jet regime, which corresponds to NAO - and $\mathrm{EA}+$, has blocks mainly over Greenland and the western Atlantic consistent with the low-frequency variability composite pattern. The northern jet regime, which corresponds to $\mathrm{NAO}+$ and $\mathrm{EA}-$, is associated with blocking anticyclones mainly over southwestern Europe. This finding is consistent with Woollings et al. (2011). The central jet regime corresponds to EA + and a neutral NAO and has a reduced frequency of occurrence of blocks, which is consistent with the reduced low-frequency variability. This suggests that the jet regimes are a good indicator of the propensity of blockings over the Atlantic.

\section{c. Regime imprint on Rossby wave breaking}

Now we examine the Rossby wave breaking characteristics of the three regime states (Fig. 4). The southern jet regime is associated with a decrease in LC1-type wave breaking equatorward of the jet stream and an increase in LC2-type wave breaking poleward of the jet stream in the North Atlantic region. The northern jet regime displays the opposite characteristics, an increase of LC1-type and a decrease of LC2-type wave breaking in the North Atlantic region. In contrast the central jet regime is associated with an increase of both LC1- and LC2-type wave breaking. There is a pronounced LC1 wave breaking increase over the subtropical North Atlantic to the east of Mexico and Florida and over the central Pacific. LC2-type wave breakings increase in a band stretching from northeastern Canada over the

\footnotetext{
${ }^{4}$ Note the different polarity in the EA in Woollings et al. (2010) and Scherrer et al. (2006). Here we use the polarity of the EA pattern as in Woollings et al. (2010).
} 


\section{a) LC1}

\section{b) LC2}

Southern Jet
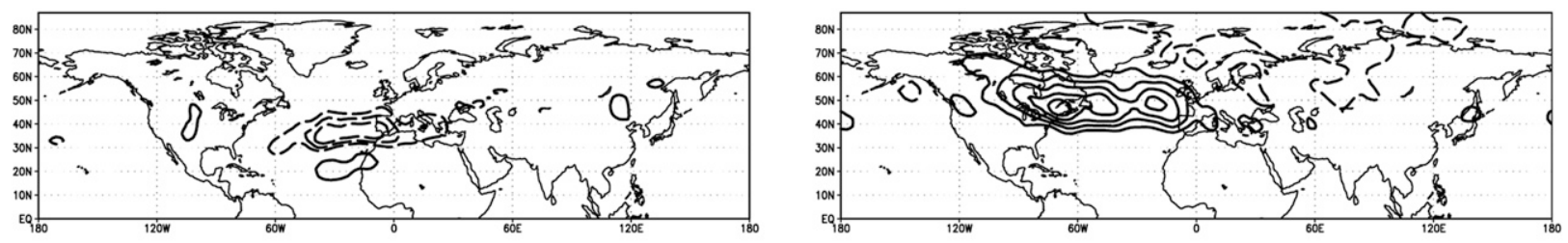

Northern Jet
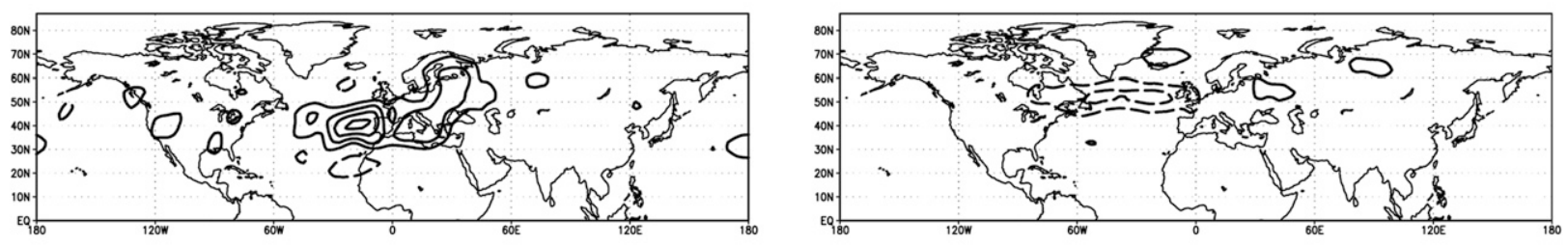

Central Jet
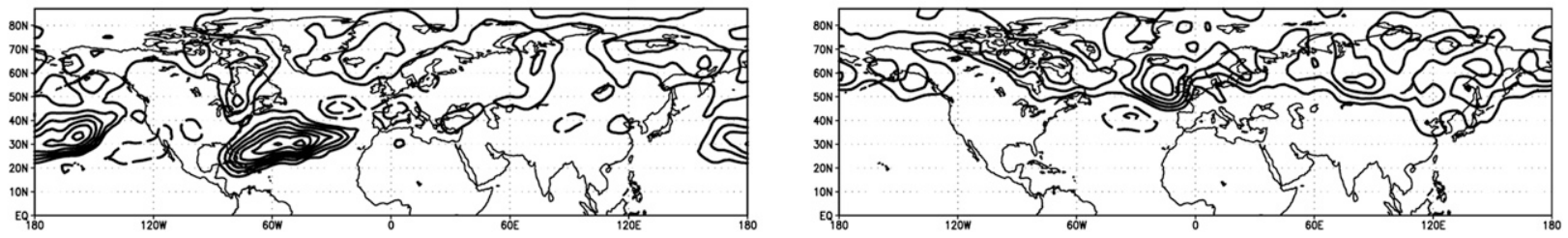

FIG. 4. PV streamer conditional distribution-(a) LC1 and (b) LC2-vertically averaged over the 310-360-K isentropes for the three different regime states. Climatological mean is subtracted. Contour interval is 0.02 .

North Atlantic and the British Isles to Siberia. The streamer distribution of the northern jet state is consistent with the streamer distribution of the positive and the streamer distribution of the southern jet state with the negative NAO [see Fig. 6 of Martius et al. (2007)]. The central jet regime is associated with reduced variability (Fig. 3) but also with enhanced LC1 breaking to the south of the jet and enhanced LC2 breaking to the north of the jet (Fig. 4). Furthermore, the maximum in LC2 wave breaking is collocated with the localized maximum of the high-frequency variability (cf. Figs. 3 and 4). The lack of variability in the subtropics of the central jet regime, where a maximum of PV streamers is found, can have two reasons. One is that the geopotential height fields do not represent subtropical variability well. Secondly, subtropical LC1 type breaking waves typically form along the eastern flank of quasi-stationary subtropical anticyclones and the variability signal could be dominated by the anticyclone and thus not appear in high-frequency variability fields. Because the central jet regime corresponds to an undisturbed jet (Woollings et al. 2010), less variability is to be expected and the observed wave breakings just maintain the undisturbed jet. That the wave breaking frequencies are enhanced relative to the climatology is consistent with the jet being stronger and sharper in the central jet regime than in the climatology (Woollings et al. 2010).

\section{d. Regime duration}

In Fig. 5 we display the regime durations. The northern jet regime is the most persistent; events can last up to 20 days. The southern jet state is the second most persistent, with states lasting up to 17 days, and the central jet regime is the least persistent, where regimes last only up to 10 days. To estimate the regime duration uncertainty we use a block bootstrap. We randomly sample from the hidden state sequence blocks 50 days in length with replacement (our results are robust to changes in the block length). We do this 1000 times and then calculate the $2.5 \%$ and $97.5 \%$ confidence levels. This procedure reveals that the central regime has a significantly shorter duration than both the northern and southern regimes. The southern and northern jet regimes have some overlap up to 6 days and for events longer than 15 days due to sampling variability. Only between 6 and 15 days are the differences in regime duration unlikely to stem from 


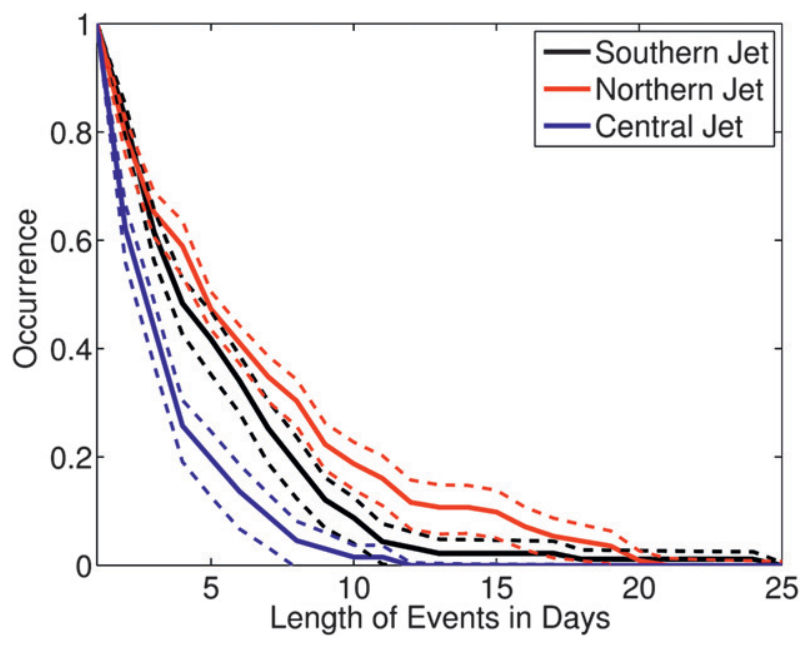

FIG. 5. Regime duration curves of the three jet regimessouthern (black), northern (red), and central (blue)—corresponding to the embedded JLI time series, expressed as the frequency of occurrences lasting at least $n$ days. The dashed curves denote the corresponding $2.5 \%$ and $97.5 \%$ confidence levels from a block bootstrap with block length of 50 days.

sampling variability (see Fig. 5). The dependence of the persistence on the regime state offers the potential of improved predictions.

It is interesting that these persistence results appear to disagree with those of Barnes et al. (2010). They suggest that in general jets are more persistent at equatorward latitudes than at poleward latitudes, and that this is true to some extent in the North Atlantic in the ERA-40 data (Barnes and Hartmann 2010). This difference could arise through differences in the methodology or in the use of all seasons here compared to winter-only in Barnes and Hartmann (2010), especially given the seasonal variation of our regime loading as described in section 7 .

\section{Preferred regime transitions}

The hidden state sequence is now examined for preferred transitions between regimes. For this purpose we compute a Markov transition matrix by considering only transitions to another regime and neglecting all dates when the system stays in the same state as in Franzke et al. (2009). This gives the following transition matrix:

$$
\mathbf{M}=\left(\begin{array}{ccc}
0 & 0.246 & \mathbf{0 . 7 5 4} \\
\mathbf{0 . 9 5 3} & 0 & 0.047 \\
0.011 & \mathbf{0 . 9 8 9} & 0
\end{array}\right)
$$

which indicates the following transition probabilities:

$$
\left(\begin{array}{lll} 
& S \rightarrow N & S \rightarrow C \\
N \rightarrow S & & N \rightarrow C \\
C \rightarrow S & C \rightarrow N &
\end{array}\right)
$$

The transition probabilities can be considered to be preferred transitions if their probability is significantly larger than when all transitions would be equally likely (i.e., the transition probability is 0.5 ). To compute significance levels for the transitions we use the approach of Horenko et al. (2008) and Franzke et al. (2009), which takes account of the different population sizes of the transitions. We claim to have preferred transitions if the transition probability and its confidence intervals are larger than 0.5. Boldface values in transition matrix (1) are significant at the $95 \%$ level. One preferred transition is from the southern to the central jet, a second is from the northern to the southern jet, and a third from the central to the northern jet. By examining Fig. 3 one can see that this corresponds to a preferred transition cycle with a northward movement of the jet. This might be the same phenomenon as described in Riehl et al. (1950), James and Dodd (1996), Feldstein (1998), and Lee et al. (2007). The transition into the southern jet regime is particularly dramatic, comprising a shift from the northern regime rather than the geographically closer central regime.

The existence of preferred transitions is consistent with the PDF of the tendencies displayed in Fig. 2. This figure displays all tendencies and is thus dominated by the small tendencies associated with the persistence property of the regime states. The occasions when the JLI transitions into another state are relatively few. The PDF is in fact heavily skewed (the skewness is -0.68). This skewness is likely due to the abrupt transitions from the northern to southern state. The skewness is not so obvious to the eye because the large negative tendencies associated with the northern to southern transitions are right in the tail of the PDF.

\section{a. Spatial evolution of preferred regime transitions}

Now we examine the evolution of the regime transitions in detail. We start with the southern to central jet transition. Lag 0 days corresponds to the date when the hidden state sequence switches to the next regime state. At lag -10 days the flow fields strongly resemble the southern jet state (Fig. 6a). Over the next few days the positive height anomaly in the north gradually weakens whereas the negative anomaly gradually moves poleward and eastward until it arrives at its final location at about lag -2 days. Over the same time period an equatorward positive anomaly gradually strengthens and propagates slowly poleward, and at lag 0 days the geopotential field looks very similar to that of the central state.

The next transition we examine is the northern to southern jet transition. Again at lag -10 days the geopotential height field strongly resembles the northern jet state (Fig. 6b). Over the next 6 days the negative 
a) $\mathrm{S} \rightarrow \mathrm{C}$
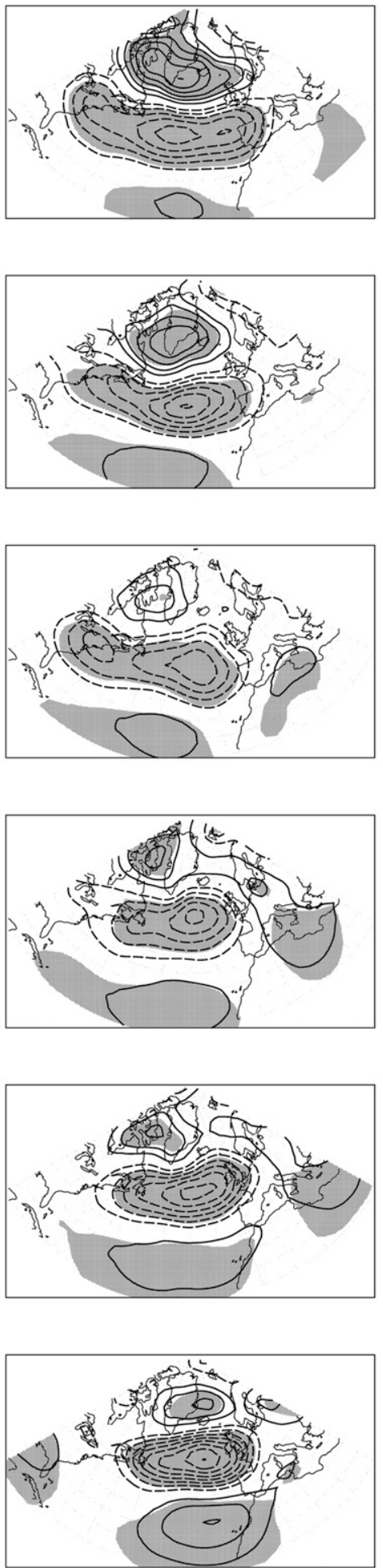

b) $\mathrm{N} \rightarrow \mathrm{S}$

\section{Lag -10 Day}

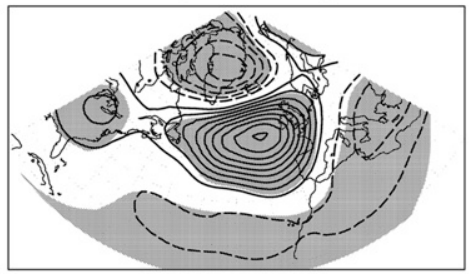

Lag -8 Day

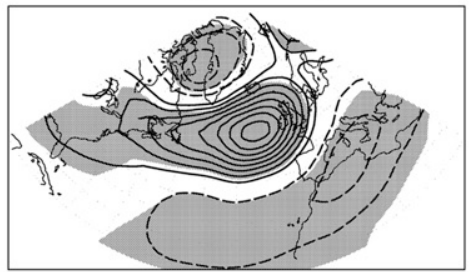

Lag -6 Day

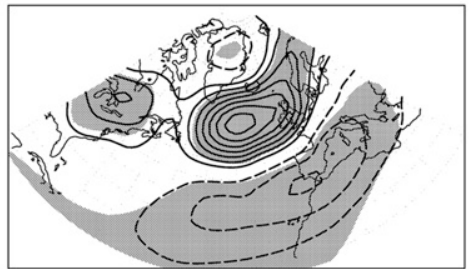

Lag -4 Day

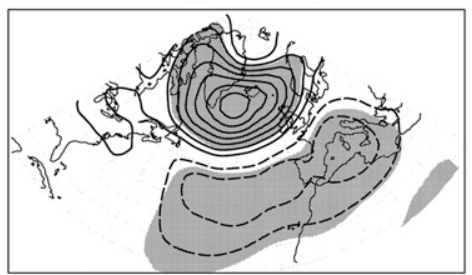

Lag -2 Day

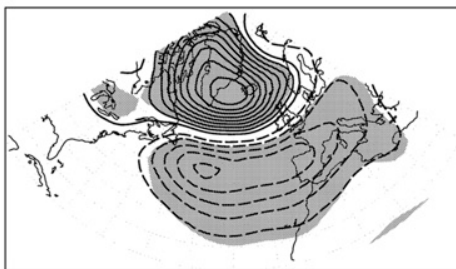

Lag 0 Day

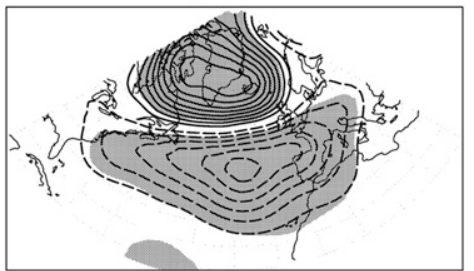

c) $\mathrm{C} \rightarrow \mathrm{N}$
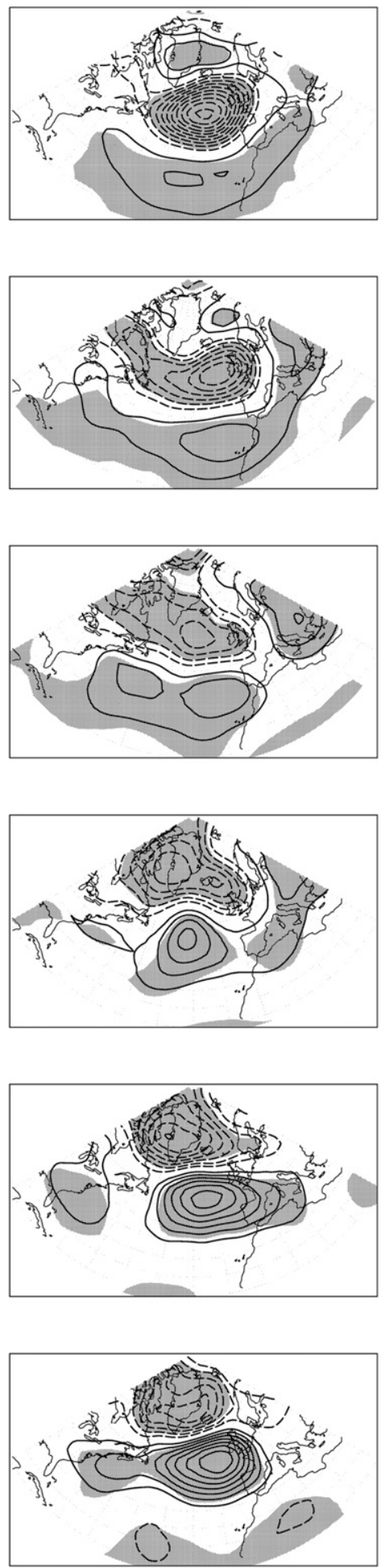

FIG. 6. Composite 500-hPa geopotential conditional mean fields for the three transitions-(a) southern to central, (b) northern to southern, and (c) central to northern-with the annual cycle subtracted. Contour interval is $100 \mathrm{gpm}$. Shaded regions are significant at the $95 \%$ level. 


\section{a) $\mathrm{S} \rightarrow \mathrm{C}$}

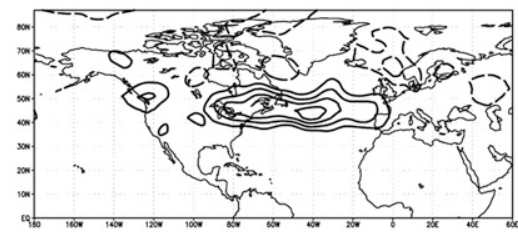

c) $\mathrm{C} \rightarrow \mathrm{N}$

Averaged Lag -10 through -7 Day
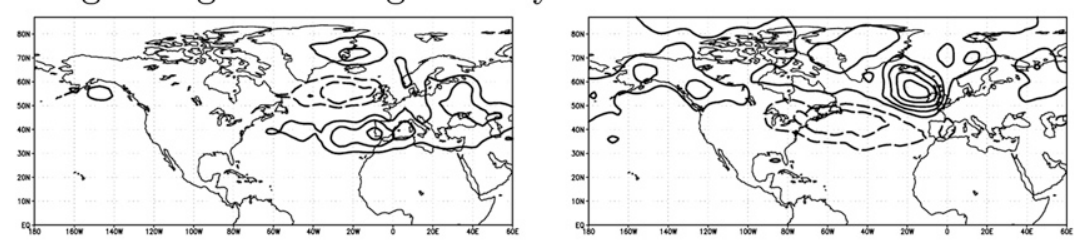

Averaged Lag -6 through -3 Day
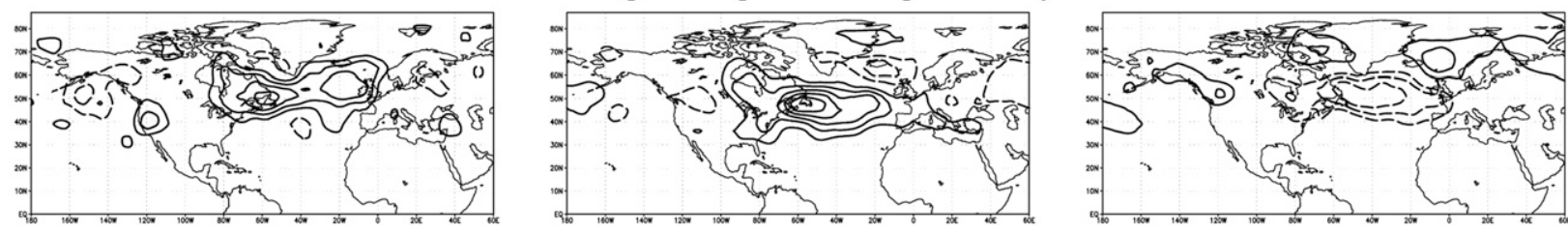

Averaged Lag -2 through +1 Day
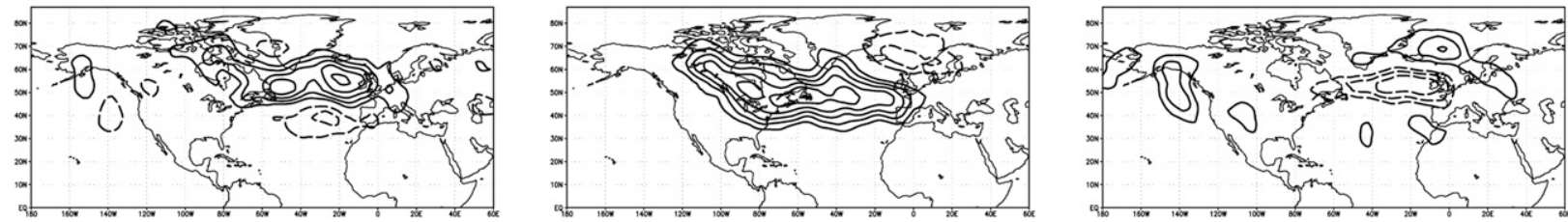

FIG. 7. LC2 PV streamer conditional distribution vertically averaged over the 310 - to $360-\mathrm{K}$ isentropes and averaged over the 4-day interval lag -2 days to lag +1 day for the three preferred regime transitions-(a) southern to central, (b) northern to southern, and (c) central to northern-with the climatological mean subtracted. Contour interval is 0.02 .

northern anomaly gradually weakens and the positive anomaly moves poleward. At the same time a negative anomaly moves from the subtropics toward the central North Atlantic and gradually strengthens until the structure of the geopotential fields is very reminiscent of the southern state.

In the central to northern state transition the state at lag -10 days strongly resembles the central state (Fig. 6c). Over the next few days the negative monopole anomaly weakens and propagates northwestward. At the same time the equatorward positive anomaly strengthens and moves poleward. This positive anomaly is initially zonally elongated and becomes more and more localized. At lag 0 days the geopotential field strongly resembles the northern state. This shows that all three preferred transitions occur on rather short time scales of less than 10 days.

\section{b. Wave breaking characteristics of preferred regime transitions}

The spatial distributions of LC1 and LC2 vary considerably in the time period prior to the regime transition and point to the central role that wave breaking plays in the regime transitions (Figs. 8 and 7, respectively). To enhance the signal to noise ratio of the frequency distributions of LC1 and LC2 we averaged over the 4-day periods of (a) lag -10 days to lag -7 days, (b) lag -6 days to lag -3 days, and (c) lag -2 days to lag +1 day.

Prior to the transition of the jet from its southern to central location, a northward shift in the location of LC2 wave breaking takes place (Fig. 7a). This northward shift is especially pronounced in the eastern Atlantic where the frequency maxima shift by approximately $10^{\circ}$ in the 10-day period prior to the jet shift. The changes in the distribution of LC1 wave breaking are relatively weaker but reveal a general increase in LC1 occurrence across the Atlantic south of $50^{\circ} \mathrm{N}$. This is clearest in the weakening of the negative anomalies at $35^{\circ} \mathrm{N}$ and the strengthening of the positive anomalies at $25^{\circ} \mathrm{N}$.

The shift of the jet from the northern to the southern position is preceded by a distinct evolution in the frequency of LC2-type events in the Atlantic basin. The spatial distribution of LC2-type events changes significantly from a local maximum over the Mediterranean 7-10 days prior to the jet shift to the formation of a frequency maximum over the western Atlantic, finally reaching a state where LC2 events are anomalously frequent across the entire North Atlantic basin (Fig. 7b). There is a significant decrease in the number of LC1 events over the western Mediterranean and eastern subtropical Atlantic in the time period prior to the 

a) $\mathrm{S} \rightarrow \mathrm{C}$

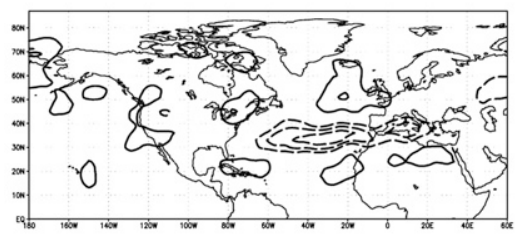

\section{c) $\mathrm{C} \rightarrow \mathrm{N}$}

Averaged Lag -10 through -7 Day
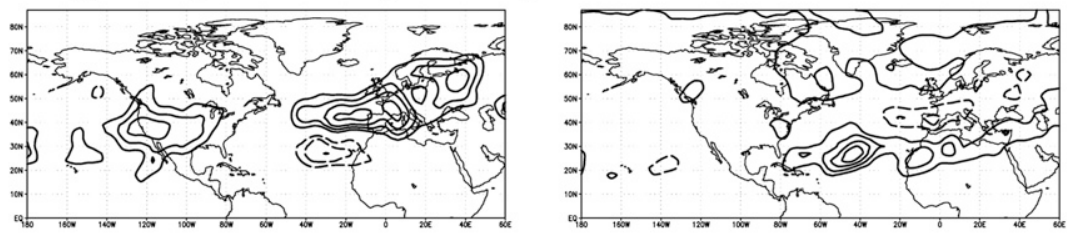

Averaged Lag -6 through -3 Day
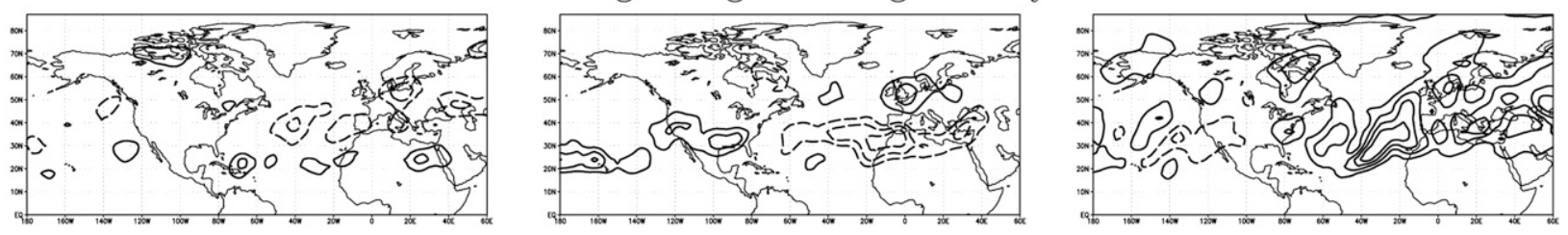

Averaged Lag -2 through +1 Day
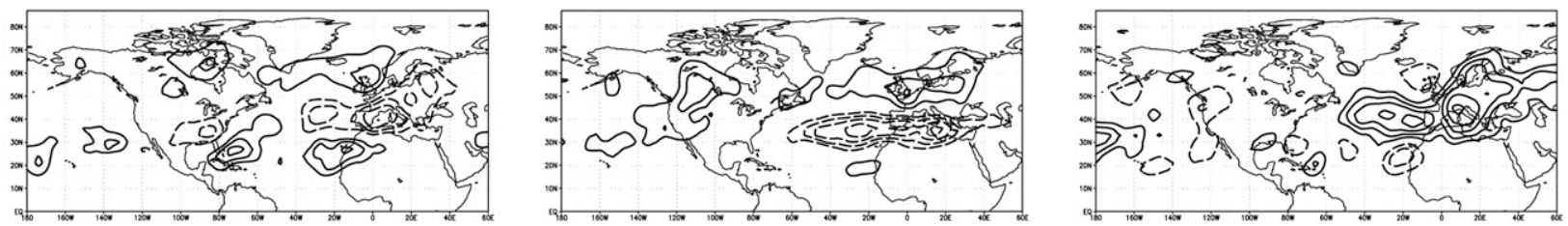

FIG. 8. As in Fig. 7, but for LC1.

southward shift of the jet. A positive frequency anomaly over western Europe weakens and shifts northward (Fig. $8 b)$. Furthermore, this transition has enhanced LC1 over the United States and enhanced LC2 over Europe and the central North Atlantic at the initial stage. These features are not visible in the northern jet composite (Fig. 4) and could be precursors to this transition.

The shift of the jet from the central to the northern position is preceded by a northward shift of at least $10^{\circ}$ of a local positive anomaly in the LC2 frequency over the eastern North Atlantic and a concomitant northward shift of a negative anomaly located farther south across the entire Atlantic basin (Fig. 7c). The concomitant changes in the LC1 anomaly patterns are slightly more complex. During the 10-day period prior to the jet shift the LC1 frequency anomalies change from a pattern with a negative anomaly over western Europe and positive anomalies in the subtropical central and eastern Atlantic, a typical central jet configuration, to a single positive anomaly in the central Atlantic and finally to a positive anomaly over the eastern north Atlantic and Europe (Fig. 8c). This transition has a negative LC2 anomaly over the North Atlantic which is not present in the central jet composite. This reduction in LC2 streamers point to the potential role RWB plays in this regime transition. As in the other transitions during the stages shown in Figs. 7b,c and
$8 b, c$, the streamer fields gradually evolve into the future jet streamer composites.

Our results highlight that individual wave breaking events are involved in the regime transitions. RWB characteristics are clearly changing prior to the northern jet to southern jet regime transition and for the central jet to northern jet regime transition. The attendant momentum fluxes will play an important role in the regime transitions.

\section{Prediction of regime states}

Because the regime states are closely related to blocking states it is potentially useful to be able to predict them. If it turns out that a simple prediction model based on the Markov transition matrix is skillful it could be used to predict the onset and decay of blockings in a complimentary fashion to state-of-the-art weather prediction models.

The temporal evolution of the regime state sequence can be described with the following deterministic equation:

$$
\pi\left(t_{0}+\tau\right)=\pi\left(t_{0}\right) \mathbf{P}^{\tau}
$$

where $\mathbf{P}$ is the Markov transition matrix (with the diagonal terms retained), $\pi$ is a discrete probability density function of the regime states, and $\tau$ is the prediction horizon (days). Equation (3) can be used for the 
prediction of regime states at some later time $t_{0}+\tau$ as in Franzke et al. (2009).

We perform prediction experiments with (3) with a 1000-member ensemble. The predictions show that our simple prediction model has a prediction horizon of about 6 days ( $e$-folding time scale) for predicting the hidden states (Fig. 9). For large prediction horizons the prediction values approach the stationary distribution of the Markov chain, which is the climatological occupancy frequency of the hidden states. The prediction horizon has two potential sources: (i) one from the metastability of the system and (ii) one from being able to predict the transition from one regime state to the next. To examine which contribution is more important we evaluate the predictive performance of these two contributions separately. As can be seen in Fig. 9, the length of the prediction horizon stems mainly from the metastability of the system (i.e., the persistence of the regime states), while the regime transitions contribute less to the overall predictability. This is not surprising because our method looks for metastable flow regimes. Our results are consistent with the prediction study by Frame et al. (2011) that analyses the skill of ensemble forecasting systems in predicting transitions between the different jet regimes of Woollings et al. (2010). Frame et al. (2011) find positive predictive skill of Atlantic jet stream transitions with about 5-10 days of lead time. Similarly, operational high-resolution ensemble prediction systems have a prediction horizon for Atlantic blockings 6-10 days ahead (Pelly and Hoskins 2003; Matsueda 2009). Hence, our computationally very cheap model has comparable predictive skill because some of our regimes are closely related to blocking.

The fact that the prediction of regime transitions contributes only a small amount to the overall predictability is not inconsistent with the existence of preferred regime transitions. In computing the transition matrix (1) we neglected all times when the system remained in the same state. This is the case for the vast majority of times because our method searches for persistent states. Thus, the number of regime transitions is rather low when compared with the number of days when the JLI stays in the same state. Thus, it is not surprising that the persistence property dominates the predictability.

\section{Annual cycle and interannual variability}

Now we discuss the annual cycle, interannual variability, and trend characteristics of the occurrence frequency of the regimes. Before the HMM analysis we subtracted the annual cycle, and thus any indication of an annual variation of the regime frequency of occurrence would be the imprint of a nontrivial annual cycle and would suggest that the JLI is nonstationary.

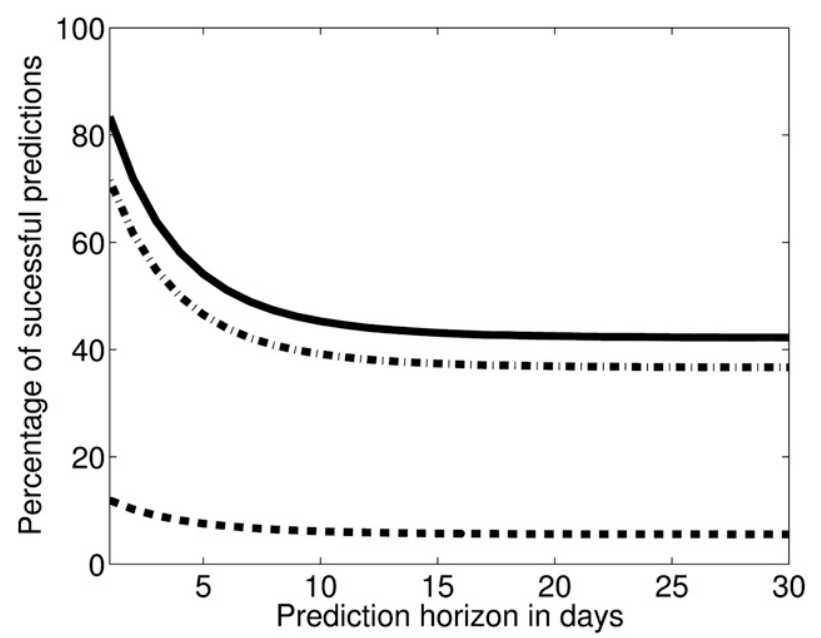

FIG. 9. Prediction skill score for predicting hidden states based on the Markov transition matrix. Solid line: total predictions; dashed line: predictions of regime switch; dashed-dotted line: prediction of persistence.

Figure 10a shows the frequency of regime occurrences through the 12 months of the year. The northern jet state is almost constant through the year and is also the most frequently visited state. The southern and central jet regimes show more variability throughout the year. Most of the time the southern jet occurs more often then the central jet; only during the summer months is there a preference of the central over the southern jet. The fact that both the northern and southern jet states occur more frequently than the central jet state is consistent with the probability distribution. As Fig. 1 reveals, the distributions of the northern and southern jet regimes are much broader then the central jet state distribution with a similar amplitude. The enhanced frequency of occurrence of the northern and central jet states during summer and autumn could reflect the seasonality of Atlantic blocking, which occurs much less often during summer than during winter (see, e.g., Scaife et al. 2010; see also our Fig. 1).

To estimate the statistical significance of the annual cycle of regime occurrence we carry out 10000 simulations with the Markov transition matrix of the HMM and then compute the 10th and 90th percentiles. The occurrence of frequency values outside of the 10th and 90th percentiles cannot be explained by sampling variability of a Markov chain and is an imprint of the annual cycle in the regime frequencies. The southern jet regime shows deviations from the ensemble in spring and summer, the northern jet regime has a higher than expected occurrence frequency during November and December, and the central jet regime has a higher occurrence frequency during summer and a lower one during November and December. These significant deviations occur even though the annual cycle of the JLI has been subtracted 
a)

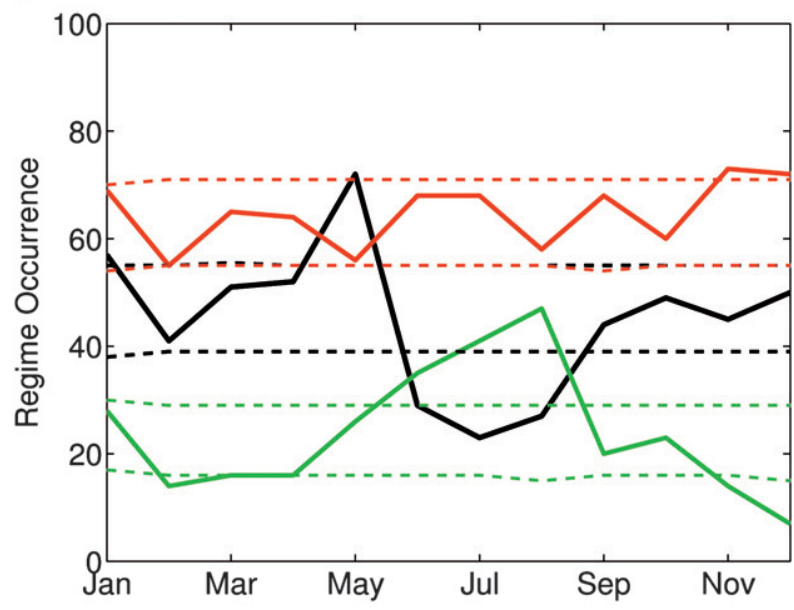

b)

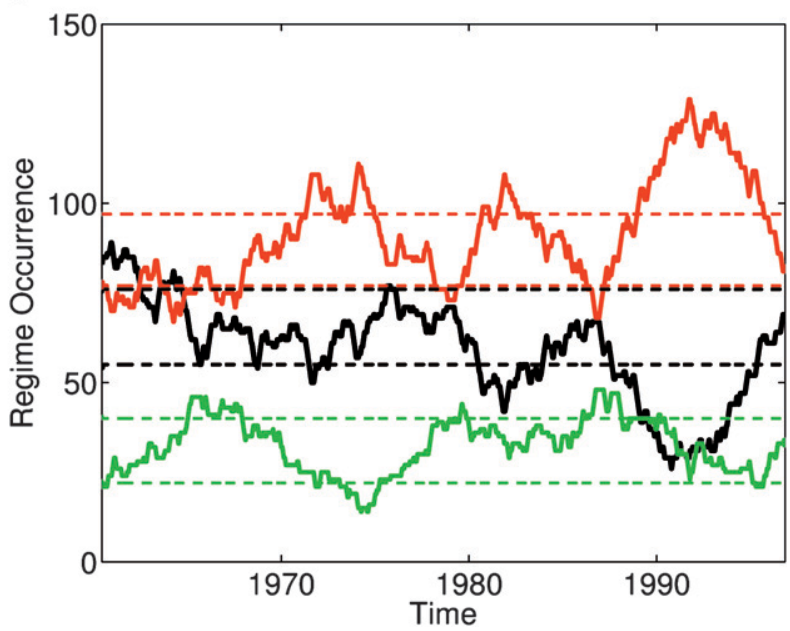

FIG. 10. (a) Annual cycle of total number of regime occurrences for the southern (black), northern (red), and central (green) jet regimes. The dashed lines denote the 10th and 90th percentiles of 10000 Markov chain simulations. (b) The 5-yr running average of hidden state occurrence [regimes and colors as in (a)]. The dashed lines denote the 10th and 90th percentiles of a 10000 member ensemble of Markov chain simulations.

before the HMM analysis. This suggests that the JLI exhibits seasonal variability in higher moments.

To examine the interannual variability characteristics we compute a 5-yr running mean of the regime occurrence frequencies (Fig. 10b). The regime occurrence frequencies undergo decadal-scale variations and there is also a hint of a preference toward the northern jet state and a corresponding decrease in southern jet regime occurrences, in agreement with Monahan et al. (2003). Especially in the 1990s the northern jet state dominated. This is consistent with the study by Franzke and Woollings (2011) that found a significant trend associated with a poleward shift in the JLI and also with the projected poleward shift of the jet stream in
Intergovernmental Panel on Climate Change (IPCC) scenario climate model projections (Yin 2005; Lorenz and DeWeaver 2007). In the late 1990s, however, the northern jet occurrence frequency decreased again and concomitantly the southern jet occurrence frequency increased. Interannual and decadal variability in the regime occurrence frequency has also been reported by Monahan et al. (2003). They attribute part of this interannual- and decadal-scale variability to the influence of the El Niño-Southern Oscillation phenomenon but suggest that a large fraction is due to chaotic internal fluctuations. This is in agreement with Franzke and Woollings (2011), who estimated that, depending on season, around $40 \%-70 \%$ of the interannual variability in the JLI is consistent with climate noise.

The annual cycle and decadal-scale variability of frequency of occurrence suggest that the JLI exhibits nonstationary regime behavior. As stated above, the seasonal cycle is subtracted from the JLI. The JLI also exhibits a poleward trend (Franzke and Woollings 2011) but repeating the regime analysis with a detrended JLI does not change the regime behavior and its interannual variability (not shown). Hence, this behavior suggests that there is substantial seasonal and interannual variability in the regime occurrence frequencies, which needs to be taken into account in future studies.

\section{Summary}

We applied the hidden Markov model method to an index of North Atlantic jet stream variability and identified three significant persistent regime states. These regime states correspond to southern, central, and northern jet states, consistent with the results of Woollings et al. (2010). These regime states are persistent states and not just recurrent states. That the jet stream regimes originally identified by Woollings et al. (2010) are found to be persistent is to be expected because the eddies driving the jet stream act in such a way as to maintain the jet in its displaced position (Branstator 1995; Lorenz and Hartmann 2003; Robinson 2006; Gerber and Vallis 2007). This positive feedback process makes the zonal wind anomalies persistent. This is also consistent with our RWB results. The regime states are accompanied by distinct changes in the storm track and low-frequency waves and frequencies of occurrence of the two types of Rossby wave breaking, anticyclonic (LC1) and cyclonic (LC2). The northern to southern jet transition is preceded by a marked increase in LC2-type wave breaking. The central to northern jet transition is preceded by an increase in LC1-type wave breaking in the central Atlantic region.

Furthermore, we identified preferred transitions between these states. The three preferred transitions are 
southern to central jet, northern to southern jet, and central to northern jet. Similar preferred regime transitions have been found by Vautard (1990), Kimoto and Ghil (1993), and Crommelin (2004). Because of different methodologies and different numbers of regimes it is hard to compare these preferred transitions in detail. In the present study the preferred jet stream regime transitions describe a preference for the poleward movement of the jet stream. This is consistent with the preferred transitions in Kimoto and Ghil (1993), which show evidence for a poleward movement of geopotential height anomalies. The preferred poleward movement of the regimes and the role played by wave breakings in the regime transitions is consistent with the mechanism put forward by Lee et al. (2007). They find in model experiments that wave breaking and linear Rossby wave propagation with a relaxation process drive the poleward zonal mean flow motion. This suggests that a feedback process is taking place. Observational evidence for such a feedback process has been found by Feldstein (1998). The preferred transition into the southern jet regime is particularly dramatic, comprising a shift from the northern rather than the central regime.

Low-frequency flow variations have been linked to changes in the occurrence of Rossby wave breaking, with wave breaking acting as a positive feedback that amplifies and maintains jet stream shifts (e.g., Riviere 2009). Further evidence for a link between synoptic-scale eddies and regimes has been found by Straus (2010). We find distinct changes in the frequency of occurrence in the $\mathrm{LC} 1$ and LC2 distributions during the regime transitions. This is particularly clear for the northern to southern and central to northern jet transitions. These results suggest that breaking waves are involved in the transition to a different regime state, and not just in the maintenance of regimes. The changes in the $\mathrm{LC} 1$ streamer distribution for the transition from the northern to the southern regime (Fig. 8) is consistent with the transition from the positive to the negative NAO as found in Martius et al. (2007). Also, the final state of this transition as seen in the LC2 streamer distribution (Fig. 7) is consistent with the LC2 streamer distribution of the negative NAO [see Fig. 6 of Martius et al. (2007)]. Taken together, our results suggest a preference for poleward jet propagation associated with LC1 wave breaking. From the northern regime the jet is then most likely to make a rapid transition directly to the southern regime, associated with LC2 wave breaking.

The transition into the northern jet regime sheds some light on different kinds of wave breaking. Woollings et al. (2011) analyzed events related to this regime, concluding that the occurrence of wave breaking acted to amplify the anomalies rather than initiate them. The difference arises because Woollings et al. (2011) used a blocking index to identify persistent wave breaking events, while there is no persistence criterion in the wave breaking method used here. This suggests that the wave breaking seen to be involved in the transition to the northern state is more transient, as in the classical LC1 paradigm of Thorncroft et al. (1993).

We also find some subtle nonstationarities in the regime occurrence frequency. The regime occurrence frequency undergoes an annual cycle and interannual variability. This is despite the fact that the annual cycle is subtracted from the JLI and no enhanced power at annual frequency is visible in a power spectral analysis of the JLI. Thus, a priori the JLI appears to be stationary. The fact that the frequency of regime occurrence shows evidence of nonstationarities means that these nonstationarities are rather subtle. The identification of the causes of these nonstationarities and how they might impact predictability needs further research. The HMM assumes stationarity of the transition process. As Fig. 10 shows, this seems not to be the case. But this is unlikely to influence the locations of the regime patterns; this is confirmed by the fact that our results are very similar to those of Woollings et al. (2010), who use methods to extract the regimes that ignore the temporal evolution of the system. However, it might influence the preferred transitions in the sense that some of the preferred transitions are more dominant in certain seasons. But the fact that our study finds a preferred poleward motion that is consistent with previous studies (Riehl et al. 1950; James and Dodd 1996; Feldstein 1998; Lee et al. 2007) suggests that our results are reliable and robust.

A possible contributing cause of the interannual variability is ocean variability (e.g., Atlantic multidecadal oscillation). An important question is how to distinguish this variability on interannual through decadal time scales from changes in weather regimes brought about by climate change. Studies by Corti et al. (1999), Palmer (1999), and Branstator and Selten (2009) provide evidence for the likely impact of climate change on the atmospheric regime structure. It will be crucial to disentangle these two effects on regimes in order to provide skillful extended-range weather and climate predictions for the coming decades.

Acknowledgments. We thank Adam Monahan and two anonymous reviewers for their comments, which helped to improve an earlier version of this manuscript. This study is part of the British Antarctic Survey Polar Science for Planet Earth Programme. It was funded by The Natural Environment Research Council. We thank ECMWF for providing the ERA-40 reanalysis data. 


\section{APPENDIX A}

\section{Hidden Markov Models}

An HMM is designed to describe a system from which only partial information is available. For example, consider a variable $Y$ that is explicitly observed; in this study this is the JLI. Now we assume that the statistics of $Y$ depend on the state of some unobservable, and therefore hidden, variable $X$. The hidden variable $X$ corresponds to the atmospheric flow regimes that determine the actual state of the JLI. In an HMM, the temporal evolution of $X$ is governed by a Markov chain. Thus, one underlying assumption of the HMM method is that the hidden state transitions are a stationary process. Furthermore, $X$ is a discrete variable and indicates to which regime state the JLI belongs at a given time $t$. The distributions of $Y$ depend on the state $X$ and we assume that they are Gaussian. As recently shown by Majda et al. (2006) and Franzke et al. (2008), the output distributions of the different hidden states can have significant overlap. Thus, the result is that the effective distribution of $Y$, which is a weighted mixture of the individual output distributions, does not need to be multimodal. A tutorial on HMMs is provided by Rabiner (1989).

An implicit assumption of the HMM methodology is that the hidden state sequence is Markovian. This is not necessarily a priori the case. To ensure Markovianity of the hidden state sequence, we embed the time series (Broomhead and King 1986; Horenko 2008b; Franzke et al. 2009):

$$
\begin{aligned}
& \tilde{\mathbf{X}}(t+\tau)=\left[\begin{array}{c}
X(t) \\
X(t+1) \\
\vdots \\
X(t+\tau)
\end{array}\right], \\
& \tilde{\mathbf{X}}(t+\tau+1)=\left[\begin{array}{c}
X(t+1) \\
X(t+2) \\
\vdots \\
X(t+\tau+1)
\end{array}\right], \\
& \tilde{\mathbf{X}}(t+\tau+2)=\left[\begin{array}{c}
X(t+2) \\
X(t+3) \\
\vdots \\
X(t+\tau+2)
\end{array}\right], \ldots
\end{aligned}
$$

where $\tau$ indicates the time lag and also the embedding dimension. Following the approaches in Franzke et al. (2008, 2009), we check for Markovianity by increasing successively the embedding dimension and stop once the system becomes Markovian. The transition matrix is Markovian for an embedding dimension of 10. All following results are based on this embedding dimension.

\section{APPENDIX B}

\section{Significance Test of Number of Hidden States}

We briefly explain our significance test to decide on the number of hidden states. As in Franzke et al. (2008), a significant gap in the values of $c_{k}$ is taken as evidence of metastability ( $\mathrm{Re}$ denotes the real part of the eigenvalue). By this we mean that the ratio $\operatorname{Re}\left(c_{k}\right) / \operatorname{Re}\left(c_{k+1}\right)$ must be significantly smaller than one finds for a HMM fitted to a reference process that is known to have no regime behavior. As a reference process we use an autoregressive moving average (ARMA) process. In Franzke and Woollings (2011) it has been shown that an ARMA process fits the autocorrelation function of the JLI well. An ARMA model is a linear model and serves in this study as our reference model for identifying metastability of the JLI. For the significance test we use an ensemble of 100 realizations of ARMA processes to estimate a significance interval for the difference between the ratio of the eigenvalues of the geophysical models and the ensemble mean ratio of the ARMA ensemble. The ratios of our geophysical models are considered significant if they are less than the empirical 10th percentile of the distribution of our ARMA ensemble.

\section{REFERENCES}

Barnes, E. A., and D. L. Hartmann, 2010: Influence of eddy-driven jet latitude on North Atlantic jet persistence and blocking frequency in CMIP3 integrations. Geophys. Res. Lett., 37, L23802, doi:10.1029/2010GL045700.

$\longrightarrow,-$ D. M. W. Frierson, and J. Kidston, 2010: Effect of latitude on the persistence of eddy-driven jets. Geophys. Res. Lett., 37, L11804, doi:10.1029/2010GL043199.

Barnston, A. G., and R. E. Livezey, 1987: Classification, seasonality, and persistence of low-frequency atmospheric circulation patterns. Mon. Wea. Rev., 115, 1083-1126.

Benedict, J. J., S. Lee, and S. B. Feldstein, 2004: Synoptic view of the North Atlantic Oscillation. J. Atmos. Sci., 61, 121-144.

Berner, J., and G. Branstator, 2007: Linear and nonlinear signatures in the planetary wave dynamics of an AGCM: Probability density functions. J. Atmos. Sci., 64, 117-136.

Branstator, G., 1995: Organization of storm track anomalies by recurring low-frequency circulation anomalies. J. Atmos. Sci., 52, 207-226.

_ change. J. Climate, 22, 2639-2658.

Broomhead, D. S., and G. P. King, 1986: Extracting qualitative dynamics from experimental data. Physica D, 20, 217-236. 
Cassou, C., 2008: Intraseasonal interaction between the MaddenJulian oscillation and the North Atlantic Oscillation. Nature, 455, 523-527, doi:10.1038/nature07286.

Cehelsky, P., and K. K. Tung, 1987: Theories of multiple equilibria and weather regimes-A critical reexamination. Part II: Baroclinic two-layer models. J. Atmos. Sci., 44, 3282-3303.

Charney, J. G., and J. G. DeVore, 1979: Multiple flow equilibria in the atmosphere and blocking. J. Atmos. Sci., 36, 12051216.

Cheng, X., and J. M. Wallace, 1993: Cluster analysis of the Northern Hemisphere wintertime 500-hPa height field: Spatial patterns. J. Atmos. Sci., 50, 2674-2696.

Corti, S., F. Molteni, and T. N. Palmer, 1999: Signature of recent climate change in frequencies of natural atmospheric circulation regimes. Nature, 398, 799-802.

Crommelin, D. T., 2004: Observed nondiffusive dynamics in largescale atmospheric flow. J. Atmos. Sci., 61, 2384-2396.

Feldstein, S. B., 1998: An observational study of the intraseasonal poleward propagation of zonal mean flow anomalies. J. Atmos. Sci., 55, 2516-2529.

- 2007: The dynamics of the North Atlantic Oscillation during the summer season. Quart. J. Roy. Meteor. Soc., 133, 15091518.

Frame, T. H. A., M. H. P. Ambaum, S. L. Gray, and J. Methven, 2011: Ensemble prediction of transitions of the North Atlantic eddy-driven jet. Quart. J. Roy. Meteor. Soc., 137, 12881297.

Franzke, C., and S. B. Feldstein, 2005: The continuum and dynamics of Northern Hemisphere teleconnection patterns. J. Atmos. Sci., 62, 3250-3267.

— , and T. Woollings, 2011: On the persistence and predictability properties of North Atlantic climate variability. J. Climate, 24, 466-472.

— S. Lee, and S. B. Feldstein, 2004: Is the North Atlantic Oscillation a breaking wave? J. Atmos. Sci., 61, 145-160.

— A. J. Majda, and G. Branstator, 2007: The origin of nonlinear signatures of planetary wave dynamics: Mean phase space tendencies and contributions from non-Gaussianity. J. Atmos. Sci., 64, 3987-4003.

, D. Crommelin, A. Fischer, and A. J. Majda, 2008: A hidden Markov model perspective on regimes and metastability in atmospheric flows. J. Climate, 21, 1740-1757.

—, I. Horenko, A. J. Majda, and R. Klein, 2009: Systematic metastable atmospheric regime identification in an AGCM. J. Atmos. Sci., 66, 1997-2012.

Gerber, E. P., and G. K. Vallis, 2007: Eddy-zonal flow interactions and the persistence of the zonal index. J. Atmos. Sci., 64, 32963311.

Hartmann, D. L., and P. Zuercher, 1998: Response of baroclinic life cycles to barotropic shear. J. Atmos. Sci., 55, 297-313.

Horenko, I., 2008a: Finite element approach to clustering of multidimensional time series. SIAM J. Sci. Comput., 32, 62-83.

- , 2008b: On simultaneous data-based dimension reduction and hidden phase identification. J. Atmos. Sci., 65, 1941-1954.

_ 2010: On the identification of nonstationary factor models and their application to atmospheric data analysis. J. Atmos. Sci., 67, 1559-1574.

— S. Solaptchiev, A. Eliseev, I. Mokhov, and R. Klein, 2008: Metastable decomposition of high-dimensional meteorological data with gaps. J. Atmos. Sci., 65, 3479-3496.

James, I. N., and J. P. Dodd, 1996: A mechanism for the lowfrequency variability of the mid-latitude troposphere. Quart. J. Roy. Meteor. Soc., 122, 1197-1210.
Kimoto, K., and M. Ghil, 1993: Multiple flow regimes in the Northern Hemisphere winter. Part II: Sectorial regimes and preferred transitions. J. Atmos. Sci., 50, 2645-2673.

Kondrashov, D., K. Ide, and M. Ghil, 2004: Weather regimes and preferred transition paths in a three-level quasigeostrophic model. J. Atmos. Sci., 61, 568-587.

_ - J. Shen, R. Berk, F. D'Andrea, and M. Ghil, 2007: Predicting weather regime transitions in Northern Hemisphere datasets. Climate Dyn., 29, 535-551.

Kravtsov, S., A. W. Robertson, and M. Ghil, 2005: Bimodal behavior in the zonal mean flow of a baroclinic $\beta$-channel model. J. Atmos. Sci., 62, 1746-1769.

Lee, S., S.-W. Son, K. Grise, and S. B. Feldstein, 2007: A mechanism for the poleward propagation of zonal mean flow anomalies. J. Atmos. Sci., 64, 849-868.

Legras, B., and M. Ghil, 1985: Persistent anomalies, blocking and variations in atmospheric predictability. J. Atmos. Sci., 42, 433-471.

Lorenz, D. J., and D. L. Hartmann, 2003: Eddy-zonal flow feedback in the Northern Hemisphere winter. J. Climate, 16, 1212-1227.

— , and E. T. DeWeaver, 2007: Tropopause height and zonal wind response to global warming in the IPCC scenario integrations. J. Geophys. Res., 112, D10119, doi:10.1029/ 2006JD008087.

Majda, A. J., C. Franzke, A. Fischer, and D. Crommelin, 2006: Distinct metastable atmospheric regimes despite nearly Gaussian statistics: A paradigm model. Proc. Natl. Acad. Sci. USA, 103, 8309-8314.

Martius, O., C. Schwierz, and H. C. Davies, 2007: Breaking waves at the tropopause in the wintertime Northern Hemisphere: Climatological analyses of the orientation and the theoretical LC1/2 classification. J. Atmos. Sci., 64, 2576-2592.

Matsueda, M., 2009: Blocking predictability in operational medium-range ensemble forecasts. SOLA, 5, 113-116.

Michelangeli, P. A., R. Vautard, and B. Legras, 1995: Weather regimes: Recurrence and quasi stationarity. J. Atmos. Sci., 52, 1237-1256.

Mo, K., and M. Ghil, 1988: Cluster analysis of multiple planetary flow regimes. J. Geophys. Res., 93, 10 927-10 952.

Monahan, A. H., L. Pandolfo, and J. C. Fyfe, 2001: The preferred structure of variability of the Northern Hemisphere atmospheric circulation. Geophys. Res. Lett., 28, 1019-1022.

_ J. C. Fyfe, and L. Pandolfo, 2003: The vertical structure of wintertime climate regimes of the Northern Hemisphere extratropical atmosphere. J. Climate, 16, 2005-2021.

Palmer, T. N., 1999: A nonlinear dynamical perspective on climate prediction. J. Climate, 12, 575-591.

Pelly, J. L., and B. J. Hoskins, 2003: How well does the ECMWF Ensemble Prediction System predict blocking? Quart. J. Roy. Meteor. Soc., 129, 1683-1702.

Rabiner, L. R., 1989: A tutorial on hidden Markov models and selected applications in speech recognition. Proc. IEEE, 77, 257-286.

Reinhold, B. B., and R. T. Pierrehumbert, 1982: Dynamics of weather regimes: Quasi-stationary waves and blocking. Mon. Wea. Rev., 110, 1105-1145.

Rennert, K. J., and J. M. Wallace, 2009: Cross-frequency coupling, skewness, and blocking in the Northern Hemisphere winter circulation. J. Climate, 22, 5650-5666.

Riehl, H., T. C. Yeh, and N. E. La Seur, 1950: A study of variations of the general circulation. J. Meteor., 7, 181-194. 
Riviere, G., 2009: Effect of latitudinal variations in low-level baroclinicity on eddy life cycles and upper-tropospheric wavebreaking processes. J. Atmos. Sci., 66, 1569-1592.

Robinson, W. A., 2006: On the self-maintenance of midlatitude jets. J. Atmos. Sci., 63, 2109-2122.

Scaife, A. A., T. Woollings, J. Knight, G. Martin, and T. Hinton, 2010: Atmospheric blocking and mean biases in climate models. J. Climate, 23, 6143-6152.

Scherrer, S. C., M. Croci-Maspoli, C. Schwierz, and C. Appenzeller, 2006: Two-dimensional indices of atmospheric blocking and their relationship with winter climate patterns in the EuroAtlantic region. Int. J. Climatol., 26, 233-249, doi:10.1002/ joc. 1250 .

Smyth, P., K. Ide, and M. Ghil, 1999: Multiple regimes in Northern Hemisphere height fields via mixture modeling clustering. J. Atmos. Sci., 56, 3704-3723.

Straus, D. M., 2010: Synoptic-eddy feedbacks and circulation regime analysis. Mon. Wea. Rev., 138, 4026-4034.

Thorncroft, C. D., B. J. Hoskins, and M. E. McIntyre, 1993: Two paradigms of baroclinic-wave life-cycle behaviour. Quart. J. Roy. Meteor. Soc., 119, 17-55.

Tung, K. K., and A. J. Rosenthal, 1985: Theories of multiple equilibria-A critical reexamination. Part I: Barotropic models. J. Atmos. Sci., 42, 2804-2819.
Uppala, S. M., and Coauthors, 2005: The ERA-40 Re-Analysis. Quart. J. Roy. Meteor. Soc., 131, 2961-3012.

Vautard, R., 1990: Multiple weather regimes over the North Atlantic: Analysis of precursors and successors. Mon. Wea. Rev., 118, 2056-2081.

Wernli, H., and M. Sprenger, 2007: Identification and ERA-15 climatology of potential vorticity streamers and cutoffs near the extratropical tropopause. J. Atmos. Sci., 64, 15691586.

Wiin-Nielsen, A., 1979: Steady states and stability properties of a low-order barotropic system with forcing and dissipation. Tellus, 31, 375-386.

Woollings, T., B. J. Hoskins, M. Blackburn, and P. Berrisford, 2008: A new Rossby wave-breaking interpretation of the North Atlantic Oscillation. J. Atmos. Sci., 65, 609-626.

A. Hannachi, and B. J. Hoskins, 2010: Variability of the North Atlantic eddy-driven jet stream. Quart. J. Roy. Meteor. Soc., 136, 856-868.

, J. Pinto, and J. Santos, 2011: Dynamical evolution of North Atlantic ridges and poleward jet stream displacements. J. Atmos. Sci., 68, 954-963.

Yin, J. H., 2005: A consistent poleward shift of the storm tracks in simulations of 21st century climate. Geophys. Res. Lett., 32, L18701, doi:10.1029/2005GL023684. 